\title{
Chapter 5. Clinical course of CKD
}

- CKD usually progresses incidiously and is often asymptomatic, but starts with urine abnormalities such as microalbuminuria, proteinuria, gradual deterioration in kidney function, and eventually progresses toward end-stage kidney disease.

- Comorbidities such as hypertension, anemia, hyperkalemia, and disorder of calcium and phosphorus metabolism appear with reduced GFR.

- If etiology of CKD is not clear, it is necessary to take a careful history including medications.

- Since CKD often lacks significant symptoms, it is critical for clinicians to know the possibility of CKD. Generally it starts with urinary abnormality and then kidney function declines gradually towards end-stage kidney disease (ESKD) (Fig. 5-1). Non-dialysis CKD patients are reported to die of cardiovascular complications before they reach ESKD. A high-risk group of CKD patients develop cardiovascular disease (CVD) at a higher rate than non-CKD population and the incidence increases exponentially with the progression of CKD (illustrated by a wider arrow toward complications in Fig. 5-1).

- Urine test provides a vey useful clue to detection of CKD. Microalbuminuria or positive urine dipstick for protein allows a diagnosis of $\mathrm{CKD}$, even in the absence of reduced kidney function. Generally, proteinuria precedes reduced kidney function, so that urine test is regularly examined particularly in a highrisk group of CKD including diabetes, hypertension, and others.

- The presence of hypertension, calcium and phosphorus disorder, and anemia often help find the presence of $\mathrm{CKD}$, and these complications are frequently

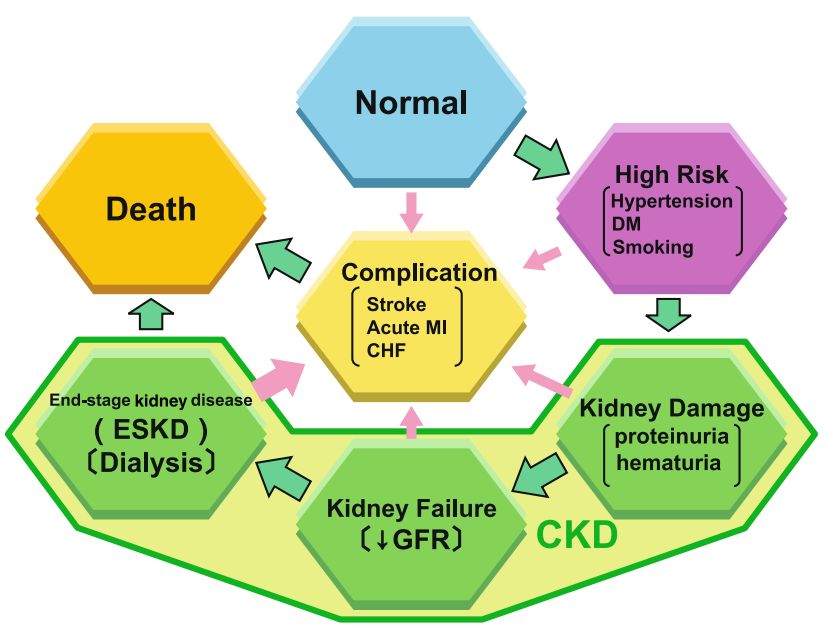

Fig. 5-1 Clinical course of CKD. CKD progresses from stage 1 to stage 5. More patients may die of cardiovascular diseases than progress to a higher stage

found in CKD stages 4 and 5 (severely reduced kidney function). It is important to make an earlier diagnosis of CKD from estimated GFR (eGFR) or urine test.

- It is noteworthy that the etiology of CKD is not identified in $9.9 \%$ of incident dialysis patients (Table 41 , see chapter 4). These patients had never visited nephrologists nor had a health checkup. They sometimes needed emergent hospitalization. The importance of regular health checkup is emphasized to the general population.

- There are cases of severely reduced kidney function (CKD stage 4-5) without a history of abnormal urine test, kidney dysfunction, nor risk factors for kidney damage. Some of those may have a drug-induced 
kidney injury, the diagnosis of which cannot be made without careful history-taking.

- In prescription of drugs excreted via kidney or with nephrotoxicity, it is recommended to evaluate and monitor eGFR. Note that many elderly people belong to CKD stage 3 (less than $60 \mathrm{~mL} / \mathrm{min} / 1.73 \mathrm{~m}^{2}$ ) despite "normal" serum creatinine level. 